\title{
Percepciones y Actitudes hacia la Violencia de Pareja contra la Mujer en Inmigrantes Latinoamericanos en España
}

\section{Perceptions and Attitudes Towards Partner Violence Against Women Among Latin-American Immigrants in Spain}

\author{
Enrique Gracia \\ Universidad de Valencia
}

\author{
Juan Herrero \\ Universidad de Oviedo
}

\author{
Marisol Lila \\ Universidad de Valencia
}

\author{
Asur Fuente \\ Universidad de Oviedo
}

\begin{abstract}
Resumen. Este trabajo analiza, en primer lugar, las percepciones y actitudes hacia la violencia de pareja contra la mujer de los inmigrantes latinoamericanos y de la población española y, en segundo lugar, explora los correlatos de las actitudes hacia la denuncia de casos de violencia de pareja contra la mujer en una muestra de inmigrantes latinoamericanos. Para ello, se utilizan 3 fuentes de datos que incorporan dos muestras representativas de la población española y un muestra de inmigrantes latinoamericanos. Los resultados muestran diferencias significativas entre españoles e inmigrantes en las percepciones y actitudes hacia la violencia de pareja contra la mujer. Los análisis también muestran que las actitudes positivas hacia la denuncia manifestadas por los inmigrantes latinoamericanos fueron más frecuentes entre aquellos que eran menos tolerantes con la violencia de pareja contra la mujer, la percibían como un problema importante en la sociedad y no tendían a culpar a las víctimas. Estos resultados subrayan la relevancia que las actitudes públicas hacia la violencia de la pareja contra la mujer tienen para la comprension y prevención de este problema social.

Palabras clave: violencia de pareja contra la mujer, actitudes, denuncia, inmigrantes

Abstract. This paper examines, first, data about perceptions and attitudes in Latin-American immigrants and the Spanish population towards intimate partner violence against women. Secondly, it explores correlates of attitudes towards reporting cases of partner violence against women in a sample of Latin-American immigrants. Three data sources, which include two representative samples of the Spanish population and a sample of Latin-American immigrants, are used. Results show significant differences between Spanish and immigrant perceptions and attitudes toward intimate partner violence against women. Analyses also show that positive attitudes among Latin-American immigrants towards reporting cases of partner violence were more frequent among those who were less tolerant, who perceived it as a major problem in society, and tended not to blame the victims. These results underline the importance of public attitudes towards intimate partner violence against women for the understanding and prevention of this social problem. Key words: partner violence against women, attitudes, reporting, immigrants.
\end{abstract}

\section{Introducción}

A pesar de ser un grupo minoritario, la violencia de pareja contra la mujer en la población inmigrante es un problema de creciente preocupación en España. Los datos oficiales sobre violencia de pareja en España muestran que un tercio de todos los casos denunciados corresponden a inmigrantes. Además, cerca del $40 \%$ de las víctimas mortales por violencia de pareja fueron mujeres inmigrantes, de las que el 50\% eran latinoamericanas (Instituto de la Mujer, 2008). Estos datos apoyan los resultados encontrados en otros contextos culturales según los cuales la violencia de pareja es una de las principales formas de victimización sufridas por

La correspondencia sobre este artículo puede dirigirse al primer autor a la Universidad de Valencia. Facultad de Psicología. Departamento de Psicología Social. Avda. Blasco Ibáñez, 21, 46010 Valencia.E-mail: enrique.gracia@uv.es las mujeres inmigrantes (e.g., Bachman y Saltzman, 1995; Davis y Erez, 1998; Dasgupta, 2000; Hazen y Soriano, 2007; Morash, Bui, Zhang y Holtfreter, 2007; Raj y Silverman, 2002).

La investigación, desarrollada mayoritariamente en EE.UU, ha revelado que la etnicidad y el estatus de inmigrante están asociados a altos niveles de participación en la violencia de pareja (Crowell y Burguess, 1996; Hass, Dutton, y Orloff, 2000; Kantor, Jasinski y Aldarondo, 1994; Raj y Silverman, 2002; Sorenson y Telles, 1991; Straus, Gelles y Steinmetz 1980; Tran y Des Jardins, 2000; Van Hightower, Gorton y DeMoss,

\footnotetext{
Agradecimientos: Este trabajo se ha realizado con la financiación de un proyecto de investigación del Ministerio de Educación y Ciencia de España (SEJ2006-08666), la Consellería de Educación de la Generalitat Valenciana (ACOMP2009/320) y el Gobierno del Principado de Asturias (PCTI 2006-2009). Una versión previa de este trabajo se publicó bajo licencia Creative Commons en The Open Family Studies Journal.
} 
2000). Los datos disponibles en España sugieren un patrón similar. La investigación disponible ha tratado de identificar los factores que podrían explicar o mediar esta relación. El estatus sociocultural, la aculturación y los roles de género (e.g., Bhanot y Senn, 2007; Jasinski, 1998; Kantor et al., 1994; Perilla, Bakeman y Rorris, 1994; West, Kantor y Jasinski, 1998); las tensiones y desafíos asociados al estatus de inmigrante (e.g., Caetano, Cunradi, Clark y Schafer, 2000; Hazen y Soriano, 2007; Rodriguez, 1998); o las percepciones de las mujeres y la tolerancia hacia la violencia de pareja (e.g., Asbury, 1993; Campbell, Maskai y Torres, 1997; Gabler, Stern y Miserandino, 1998; Gondolf, Fisher y McFerron, 1991), se han considerado factores importantes que podrían explicar el incremento del riesgo de sufrir violencia de pareja en grupos minoritarios como el inmigrante. Además, se ha sugerido que los mayores niveles de participación en la violencia por parte de minorías e inmigrantes están mediados en parte por el contexto comunitario en el que viven -ambientes muy desaventajados o de pobreza extrema, segregación, aislamiento social (e.g., Benson, Wooldredge, Thistlethwaite y Fox, 2004; Gracia y Herrero, 2007; Gracia, Herrero, Lila y Fuente, 2009; Herrero y Gracia, 2005; Sampson y Lauritsen, 1994; Wilson, 1987). Sin embargo, en su revisión, McLoyd, Cauce, Takeuchi y Wilson (2000), han puesto de manifiesto que los controles estadísticos en cuanto a ingresos, educación, entorno urbano, edad, número de hijos y duración del matriomio, reducen la relación observada entre la pertenencia a grupos minoritarios y violencia doméstica, pero no la eliminan.

Un aspecto escasamente estudiado en este ámbito de investigación ha sido, sin embargo, las diferencias en las actitudes públicas hacia la violencia de pareja entre la población nativa e inmigrante. En este estudio, en lugar de centrarnos en las actitudes de las víctimas o los agresores, nos centraremos en las actitudes de la población general (tanto nativa como inmigrante) hacia la violencia de pareja contra la mujer. En la primera parte del estudio, compararemos las percepciones y actitudes respecto a la violencia de pareja contra la mujer entre la población española y los inmigrantes latinoamericanos residentes en España. Así, compararemos, por una parte, el grado de exposición pública a incidentes de violencia de pareja (si los informantes conocen víctimas) y la frecuencia percibida de este tipo de violencia en la sociedad. Por otra parte, se compararán un conjunto de actitudes indicadoras del grado de aceptabilidad social de la violencia de pareja contra la mujer: la tolerancia hacia la violencia de pareja, la culpabilización de las víctimas y las actitudes hacia la denuncia a la policía de casos de violencia de pareja conocidos. Dada la alta incidencia de violencia de pareja en España entre inmigrantes hipotetizamos que, en comparación con la población española, los inmigrantes latinoamericanos conocerán a más mujeres que hayan sido maltratadas por sus parejas y que lo considerarán un problema más común. También hipotetizamos que entre los inmigrantes habrá un clima social más favorable hacia la violencia de pareja. Esto es, en comparación con la población española, la violencia de pareja sera más tolerada, las mujeres tenderán a ser más culpabilizadas y las actitudes positivas ante la denuncia de casos a las autoridades serán menos comunes en la población inmigrante.

En la segunda parte del estudio analizaremos la disposición de los inmigrantes latinoamericanos a ejercer el control social informal sobre la violencia de pareja, explorando los correlatos de sus actitudes hacia la denuncia de situaciones de violencia de pareja ante las autoridades. Salvo excepciones (ver Gracia y Herrero, 2006a, para un análisis de los correlatos de las actitudes de la población española hacia la denuncia de casos de violencia de pareja contra la mujer), se ha invertido poco esfuerzo investigador en la exploración de los factores que influyen en la disposición pública a actuar ante situaciones de violencia de pareja contra la mujer. Las actitudes públicas hacia la denuncia de este tipo de casos proporciona un indicador del clima social que rodea a las víctimas respecto a la violencia de pareja y de la disposición pública a ejercer el control social sobre este problema (e.g., Fagan, 1993; Ferrer, Bosch y Riera, 2006; Gelles, 1983; Gracia, 2002, 2004; Lackey y Williams, 1995). Como argumentó Fagan (1993), la disposición a ejercer control social entre las redes sociales y las comunidades en las que las parejas están inmersas puede jugar un papel disuasorio importante así como prevenir la violencia de pareja. Un ambiente social (incluyendo vecinos, amigos, compañeros de trabajo o conocidos) dispuesto a actuar e involucrarse puede inhibir a los agresores y reducir las inhibiciones tanto de los testigos como de las víctimas a la hora de denunciar (Fagan, 1993; Gelles, 1983; Kelly, 1996).

En este estudio exploraremos diversos correlatos de las actitudes hacia la denuncia de casos de violencia de pareja contra la mujer en una muestra de inmigrantes latinoamericanos: exposición a situaciones de violencia de pareja, frecuencia percibida de este problema, tolerancia y culpabilización de las víctimas. Las percepciones y actitudes en torno a la violencia de pareja pueden ser factores relevantes a la hora de determinar las actitudes de las personas hacia la denuncia. Por ejemplo, algunas investigaciones sugieren que conocer una víctima de violencia en la pareja está asociado con una actitud negativa ante la denucnia (Gracia y Herrero, 2006a). La frecuencia percibida de la violencia de pareja también puede ayudar a conformar las actitudes públicas hacia la denuncia así como incrementar el sentido de responsabilidad personal (Klein et al., 1997). Las actitudes hacia la denuncia de los casos de violencia en la pareja también pueden depender de la tolerancia pública hacia todos o solamente ciertos tipos de 
incidentes. Por ejemplo, si la violencia en la pareja contra la mujer sólo se considera como tal cuando implica violencia extrema, severa o repetida, es más probable que la violencia contra la mujer en las relaciones íntimas pueda ser aceptada en ciertas circunstancias (Loseke, 1989; Muehlenhard y Kimes, 1999). Si algunos incidentes de violencia de pareja son percibidos como aceptables o no suficientemente graves (e.g., amenazas, agresiones físicas no severas) es menos probable que aparezcan actitudes positivas hacia la intervención. Por ejemplo, la investigación ha mostrado que mayores niveles de tolerancia (e.g., pensar que algunos niveles de violencia son normales y aceptables) están asociados con actitudes negativas hacia la denuncia (Gracia y Herrero, 2006a; Gracia, García y Lila, 2009). Las actitudes de culpabilización de las víctimas también pueden influir negativamente en la actitud de denunciar. Como ha mostrado la investigación psicosocial, responsabilizar a la víctima de su propia victimización reduce sig-nificativamente las posibilidades de ofrecer ayuda (Lerner, 1970, 1980; Weiner, 1980, 1995).

Finalmente, controlamos ocho variables de tipo sociodemográfico (género, edad, estado civil, ingresos, educación, años de residencia en España, confianza en las autoridades y estatus legal como inmigrante) que podrían estar relacionadas tanto con las variables predictoras como con la dependiente (Gracia y Herrero, 2006a; Kantor et al., 1994; McLoyd et al., 2000; Stowell y Martinez, 2007; West, Kantor y Jasinski, 1998).

\section{Método}

\section{Participantes}

En este estudio se utilizan dos tipos de datos. En primer lugar, se utilizan datos disponibles de encuestas nacionales representativas de la población española sobre violencia en la pareja contra la mujer. Estos datos incluyen una encuesta nacional española con una muestra de 2.498 adultos (18 o más años) realizada en 2004 por el Centro de Investigaciones Sociológicas para abordar diferentes cuestiones sobre la violencia doméstica. También se utilizaron en el estudio datos del Eurobarómetro 51.0 "Los europeos y sus puntos de vista sobre la violencia doméstica contra la mujer", realizado con una muestra de cada uno de los estados miembros de la Unión Europea (ver Gracia y Herrero 2006a,b para una descripción detallada de esta encuesta).

En segundo lugar, participaron en el estudio 399 inmigrantes latinoamericanos que en el momento de la investigación residían en dos comunidades autónomas en España: Asturias y Valencia. Para la selección de los participantes se siguió un proceso en dos fases. En la primera, miembros del equipo de investigación contactaron con organizaciones no gubernamentales y otras asociaciones establecidas legalmente en ambas comunidades que en el momento de la aplicación de los cuestionarios trabajaban con inmigrantes latinoamericanos. Estas organizaciones cubrían un amplio abanico de actividades con inmigrantes (desde ayuda legal a actividades de ocio). La principal razón para contactar con estas asociaciones fue obtener acceso a un importante número de participantes con los requisitos necesarios para el estudio. Durante esta primera fase, se explicaron los principales objetivos del estudio a los responsables de las organizaciones y se les extendió una invitación para que sus miembros participaran en él voluntariamente. En la segunda fase, se facilitaron autoinformes a aquellos miembros que desearon participar en el estudio con instrucciones para que los cumplimentaran y los remitieran a la propia asociación.

\section{Medidas}

Exposición personal. A los participantes se les preguntó: "¿Conoce algún caso de violencia doméstica contra la mujer?" $(1=n o, 2=s \hat{\imath})$.

Frecuencia percibida. Se utilizó la siguiente pregunta: "En su opinión, ¿cuál es la frecuencia de la violencia doméstica contra la mujer en la sociedad española?" Las categorías de respuesta fueron: 1 = nada frecuente, $2=$ algo frecuente, $3=$ frecuente, $\mathrm{y} 4=$ muy frecuente.

Tolerancia. La pregunta en este caso fue: “¿En qué circunstancias considera que una mujer víctima de violencia por parte de su pareja debería denunciarlo a las autoridades?" Las categorías de respuesta fueron: $1=$ tan pronto se sienta amenazada por su pareja aunque no haya agresiones físicas, $2=$ tan pronto haya una agresión aunque no sea grave, y 3 = sólo cuando haya una agresión grave.

Culpabilización de las víctimas. Los participantes tenían que mostrar su acuerdo o desacuerdo con la siguiente afirmación: "Una causa de la violencia de pareja contra la mujer es la conducta provocativa de la mujer" $(1=s i ́, 0=n o)$.

\section{Variable dependiente}

Actitudes ante la denuncia. Esta variable fue evaluada con la siguiente pregunta: "Qué haría si estando en casa usted oye que un vecino está golpeando a su mujer?" $(0=$ no lo denunciaría a la policía $1=l o$ denunciaría a la policía).

\section{Variables de control}

La edad fue medida en años. El género se codificó en $1=$ hombre y $2=$ mujer . El nivel educativo fue categorizado en: 1 = sin estudios, 2 = estudios elementales, $3=$ bachillerato y $4=$ estudios universitarios. El esta- 
do civil: $1=$ soltero, 2 = casado, $3=$ separado/divor ciado. Los ingresos familiares se dividieron en 12 categorías, desde $1=$ menos de 1.800 euros/año a $12=$ más de 120.000 euros/año. El tiempo de residencia en España fue medido en años. Para codificar el estatus legal como inmigrante preguntamos a los participantes si estaban legalmente establecidos en el país $(1=s i ́ 2=$ no). También incluimos una variable de control adicional (confianza en la policía) para averiguar si las actitudes hacia la denuncia estaban condicionadas por una actitud previa hacia la policía. La confianza en la policía fue medida con un ítem adaptado de la European Social Survey (2008): “¿Qué confianza le ofrece la policía?" Las categorías de respuesta oscilaron entre 0 (no me ofrece ninguna confianza) y 10 (confio plenamente). En la Tabla 1 se presenta la distribución de las variables sociodemográficas y de control.

\section{Resultados}

En torno a un $40 \%$ de los participantes fueron hombres. El nivel educativo medio en el estudio fue elevado (el porcentaje de participantes con bachillerato o superior se situó por encima del $80 \%$ ). La mayoría de los participantes estaban casados en el momento del estudio $(52.9 \%)$ y se encontraban en situación regularizada $(80.4 \%)$. La edad media fue de 34 años y el ingreso medio por hogar fue bajo (6.000-12.000 euros al año). El tiempo medio de residencia en España fue de 5 años. Finalmente, los participantes informaron de una confianza en la policía moderada (6.3 sobre una escala de 10).

\section{Percepciones y actitudes hacia la violencia de pareja contra la mujer en españoles e inmigrantes lationamericanos}

En la primera parte del estudio, analizamos si los participantes españoles e inmigrantes mantenían una visión diferente sobre la violencia en la pareja contra la mujer y, también, si ambos grupos presentaban difierentes niveles de exposición a la violencia de pareja contra la mujer. Para ello, comparamos la distribución de las respuestas de la población española (contenidas en encuestas nacionales) con las respuestas de los participantes latinoamericanos del estudio. La Tabla 2 presenta la distribución de las respuestas tanto para los españoles como para los inmigrantes junto con el análisis Chi-cuadrado de las diferencias.

Los resultados de la Tabla 2 indican con claridad que en España los inmigrantes latinoamericanos mantienen visiones diferentes sobre la violencia de pareja contra la mujer en comparación con la población española y, además, que este colectivo está mucho más expuesto a este tipo de violencia. Así, el porcentaje de inmigrantes que conocía a una víctima de violencia en la pareja contra la mujer supuso más del doble que el de la población española. Quizás, como consecuencia de ello, los inmigrantes percibían una mayor frecuencia de este tipo de violencia contra la mujer en España que la población española. Finalmente, los inmigrantes toleraban la violencia en la pareja contra la mujer en mayor medida, tendían a culpabilizar más a la víctima y denunciarían casos de violencia en la pareja contra la mujer en menos ocasiones que la población española.

Tabla 1. Distribución de las variables sociodemográficas y de control $(\mathrm{N}=399)$

\begin{tabular}{|c|c|}
\hline & Porcentajes \\
\hline Hombres & 40.7 \\
\hline \multicolumn{2}{|l|}{ Nivel educativo } \\
\hline $\begin{array}{l}\text { Sin estudios } \\
\text { Educación Básica } \\
\text { Bachillerato } \\
\text { Estudios universitarios }\end{array}$ & $\begin{array}{r}0.7 \\
13.9 \\
58.6 \\
26.8\end{array}$ \\
\hline \multicolumn{2}{|l|}{ Estado Civil } \\
\hline $\begin{array}{l}\text { Soltero } \\
\text { Casado } \\
\text { Separado/Divorciado }\end{array}$ & $\begin{array}{l}34.3 \\
52.9 \\
12.8\end{array}$ \\
\hline \multirow[t]{2}{*}{ Situación Regularizada (Sí) } & 80.4 \\
\hline & Media \\
\hline $\begin{array}{l}\text { Edad } \\
\text { Ingresos en el hogar } \\
\text { Tiempo de residencia en España } \\
\text { Confianza en la Policía }\end{array}$ & $\begin{array}{c}34.6 \\
6,000-12,000 \text { euros/año } \\
5 \text { años } \\
6.3\end{array}$ \\
\hline
\end{tabular}


Tabla 2. Percepciones y actitudes hacia la violencia de pareja contra la mujer en españoles e inmigrantes latinoamericanos en España

\begin{tabular}{lccc}
\hline & Españoles $(\%)$ & Immigrantes $(\%)$ & $\chi^{2}$ \\
\hline Exposición Personal (Sí) & 23.0 & 54.1 & $170.30^{* * *}$ \\
Frecuencia Percibida & & & \\
$\quad$ Muy frecuente & 16.6 & 55.9 & $306.17 * * *$ \\
Tolerancia & & & 69.3 \\
$\quad$ Amenazas & 78.5 & 56.5 & $16.01^{* * *}$ \\
Culpabilización de la víctima (Sí) & 39.2 & 63.8 & $21.56^{* * * *}$ \\
Denunciaría (Sí) & 75.0 & &
\end{tabular}

$* * * \mathrm{p}<.001$

\section{Correlatos de las actitudes hacia la denuncia de casos de violencia en la pareja contra la mujer}

En la segunda parte del estudio, analizamos los correlatos de las actitudes hacia la denuncia a las autoridades de casos de violencia de pareja contra la mujer. Para este análisis, realizamos un análisis de regresión logística binomial multivariada, técnica indicada para variables dependientes con dos categorías de respuesta (Menard, 1995). Las variables predictoras no continuas se incluyeron en el modelo como variables categóricas con el objeto de estimar 'si el efecto de pertenecer a una categoría es diferente y estadísticamente significativo al de la categoría de referencia' (Menard, 1995 , p. 52). Para evaluar el ajuste del modelo estimamos el Chi-cuadrado asociado, que es análogo al test basado en la $\mathrm{F}$ multivariada en la regresión lineal. El rechazo de la hipótesis nula significa que al menos una beta en el modelo no es cero (Damaris, 1995). En otras palabras, que la información sobre las variables independientes permiten mejoran nuestras predicciones sobre la variable dependiente. Los análisis se llevaron a cabo utilizando el paquete estadístico SPSS 15.

En la parte superior de la Tabla 3 presentamos los resultados correspondientes a la regresión logísica binomial multivariada. El Chi-cuadrado asociado al modelo fue estadísticamente significativo $\left(\chi^{2}=66.19\right.$, $\mathrm{gl}=15, \mathrm{p}<.001)$ (ver parte inferior de la Tabla 3), lo que permite rechazar la hipótesis nula de que los correlatos de las actitudes hacia la denuncia no mostraban una asociación estadísticamente significativa con la variable dependiente.

La única variable de control estadísticamente significativa fue el género, con una dismunción en la razón de posibilidades $(\exp (\mathrm{B}))$ para los hombres frente a las mujeres del $65.1 \%((1-.349) \times 100)$. Todas las variables centrales del modelo mostraron significación estadística, con la excepción de la exposición personal a casos de violencia. Con respecto a las variables con una relación positiva con las actitudes hacia la denuncia encontramos que un incremento en una unidad en la frecuencia percibida se asoció con un incremento del $227 \%$

Tabla 3. Resultados de la Regresión Logística Binomial Multivariada para las actitudes hacia la denuncia de casos de violencia de pareja contra la mujer ${ }^{1,2}$

\begin{tabular}{|c|c|c|c|c|}
\hline & $\mathrm{B}$ & E.T. & Sig. & $\operatorname{Exp}(B)^{a}$ \\
\hline Hombres & -1.052 & .517 & .042 & .349 \\
\hline Frecuencia & 1.184 & .350 & .001 & 3.269 \\
\hline Culpabilización de la Víctima (Sí) & -.662 & .229 & .004 & .516 \\
\hline Exposición Personal (Sí) & -.764 & .508 & .132 & .466 \\
\hline Tolerancia ${ }^{b}$ & & & .001 & \\
\hline Agresión no severa & -1.623 & .525 & .002 & .197 \\
\hline Agresión severa & -2.788 & 1.071 & .009 & .062 \\
\hline \multicolumn{5}{|c|}{$\begin{array}{l}{ }^{1} \text { Chi-cuadrado del Modelo }(\mathrm{gl}=15)=66.19, \mathrm{p}<.001 ; \mathrm{R}^{2} \text { Nagelkerke }=.44 \\
{ }^{2} \text { Las variables de control no significativas son: edad, años de residencia en España, estado civil, nivel educativo, ingresos en el hogar, situación regularizada, y confianza en la policía. } \\
\text { a Exp (B) es la razón de posibilidades (odds ratio). Los valores significativos mayores que } 1 \text { indican una actitud postiva hacia la denuncia de casos de violencia de pareja contra la mujer. Valores } \\
\text { significativos menores que } 1 \text { indican una actitud negativa hacia la denuncia de casos de violencia de pareja contra la mujer. } \\
\text { b Categoría de referencia: amenazas. }\end{array}$} \\
\hline
\end{tabular}


((3.269-1)x100) en la razón de posibilidades para denunciar casos de violencia de pareja contra la mujer. En cuanto a las variables negativamente asociadas con la denuncia, encontramos un descenso significativo en la razón de posibilidades del $48.4 \%$ en aquellos que culpabilizaban a la victima frente a los que no lo hacían. Además, aquellos que expresaban que la mujer debería denunciar tan sólo cuando hubiera agresión, aunque ésta no fuera severa, redujeron su razón de posibilidades en un $80.3 \%$. Finalmente, aquellos que sólo justificaban la denuncia en presencia de agresión grave mostraron una reducción de la razón de posibilidades hacia la denuncia en un $93.8 \%$.

\section{Discusión}

Numerosos estudios han mostrado altos niveles de violencia de pareja en la población inmigrante. En España, los datos también revelan una mayor incidencia de violencia de pareja contra la mujer (con y sin resultado de muerte) en población inmigrante, en particular entre las personas procedentes de América Latina. Para mejorar nuestra comprensión del vínculo existente entre inmigración y violencia de pareja, en la primera parte de este estudio comparamos las percepciones y actitudes hacia la violencia de pareja contra la mujer de españoles e inmigrantes latinoamericanos residentes en España. La segunda parte del estudio exploró la disposición de los propios inmigrantes a ejercer el control social informal analizando diferentes correlatos de las actitudes hacia la denuncia de casos conocidos de violencia de pareja contra la mujer.

En primer lugar, los resultados muestran que la alta incidencia de violencia de pareja en inmigrantes se corresponde también con sus percepciones. En comparación con los españoles, los inmigrantes manifestaron conocer más víctimas $(54.1 \%$ vs. $23 \%)$ y percibieron la violencia de pareja como más frecuente en la sociedad ("muy frecuente" $=55.9 \%$ vs. $16.6 \%$ ). Los resultados también muestran que, en comparación con los participantes españoles, hay entre los inmigrantes latinoamericanos un clima social más favorable hacia la violencia de pareja. De acuerdo con nuestros datos, este clima social de aceptabilidad puede representarse en términos de una mayor tolerancia hacia la violencia ("la violencia de pareja debería ser denunciada a las autoridades tan pronto como la mujer sienta que está siendo amenazada, incluso si no hay agresiones físicas" $=69 \%$ de inmigrantes de acuerdo vs. $78.5 \%$ de españoles), de una mayor tendencia a culpabilizar a las mujeres víctimas $(56.5 \%$ vs. $39.2 \%)$ y de una menor disposición a ejercer control social denunciando ante las autoridades los casos conocidos de violencia de pareja contra la mujer (63.8\% vs. $75 \%)$. Estos resultados podrían contribuir a explicar las mayores tasas de violencia de pareja entre la población latinoamericana en España y apoyan la idea de que las tasas de violen- cia en la pareja íntima están relacionadas con el clima social de aceptabilidad y tolerancia (Fagan, 1989; Gelles, 1983; Gracia, 2004; Gracia y Herrero, 2006b; Klein et al., 1997). En este sentido, la Organización Mundial de la Salud (2002) consideró entre los grandes factores sociales que influyen en las tasas de violencia aquellos que crean un clima de aceptabilidad hacia la violencia y reducen las inhibiciones contra la misma. Un ambiente social que condone o incluso apoye la violencia de pareja hace más probable que los agresores persistan en su comportamiento violento (e.g., Bennet y Williams, 1999; Fagan, 1990; Harden y Koblinsky, 1999; Klein et al., 1997). Esta visión es compartida por numerosos estudiosos que creen que sin un cambio importante en las actitudes sociales que perpetúan los actos de violencia contra las mujeres el problema no será resuelto (Biden, 1993; Gracia, 2004; Gracia y Herrero, 2006b; Goodman et al., 1993; Klein et al., 1997). Como puso de manifiesto Biden (1993, p. 1060) "no podemos esperar una respuesta eficaz ante la violencia contra la mujer a menos que confrontemos y condenemos las actitudes que cultivan la violencia". A este respecto, nuestros resultados también sugieren la necesidad de una mejor educación dirigida a aquellos grupos que mantienen actitudes de aceptabilidad y tolerancia hacia la violencia de pareja, para lograr una prevención más eficaz (Gracia, 2004).

En la segunda parte del estudio exploramos los correlatos de las actitudes de los inmigrantes latinoamericanos hacia la denuncia a las autoridades en caso de conocer casos de violencia de pareja contra la mujer. Los resultados del análisis de las variables sociodemográficas mostraron que las mujeres manifiestan una actitud más positiva hacia la denuncia que los hombres. Estos resultados son acordes con la investigación disponible y sugieren que las mujeres perciben la violencia de pareja como más grave y menos acceptable y sienten más responsabilidad personal para actuar que los hombres, lo que apunta a una mayor empatía con las víctimas (Gracia, García y Lila, 2008, 2009; West y Wandrei, 2002). Ninguno de los otros controles sociodemográficos explorados produjo diferencias significativas.

Con respecto a los correlatos de las actitudes hacia la denuncia de casos de violencia de pareja contra la mujer, todas las variables examinadas, excepto la "exposición personal", mostraron significación. Esto es, entre inmigrantes latinoamericanos, aquellos que perciben la violencia de pareja como más frecuente en la sociedad, son menos tolerantes con ella y no tienden a culpabilizar a las víctimas, tienen una actitud más positiva hacia la denuncia de casos de violencia de pareja contra la mujer.

En cuanto a la frecuencia percibida de la violencia de pareja contra la mujer en la sociedad, encontramos, como en un estudio previo con población española (Gracia y Herrero, 2006a), una correlación positiva y estadísticamente significativa con las actitudes positi- 
vas hacia la denuncia. Esto sugiere que un mayor reconocimiento público de la ocurrencia de la violencia de pareja en la sociedad favorece actitudes positivas a la hora de denunciarlo a las autoridades.

Como argumentaron Klein et al. (1997), la responsabilidad social y personal para tomar parte contra la violencia de pareja proviene de creer que el problema es importante y lo suficientemente amenazante para el tejido social como para afectar la propia vida. Esto también sugiere que los esfuerzos pasados y futuros por incrementar la visibilidad social de la violencia de pareja contra la mujer han ayudado y probablemente continuarán ayudando a conformar las actitudes públicas frente al problema incrementando los sentimientos de responsabilidad (Klein et al., 1997; Straus y Gelles, 1986). Por tanto, un objetivo importante para las campañas de educación pública es continuar incrementando la conciencia pública sobre la alarmante presencia de la violencia de pareja contra la mujer en nuestra sociedad.

Nuestros resultados también mostraron que un menor nivel de tolerancia hacia la violencia se asocia con actitudes positivas hacia la denuncia. Por otra parte, muchos participantes parecen tolerar en mayor grado ciertos niveles de violencia (ej.: amenazas, agresiones físicas no severas). En línea con otros estudios, este resultado sugiere que cuando cierto nivel de violencia hacia la mujer (excepto formas extremas) es percibido como más "tolerable", las posibilidades de que los otros sean sensibles a las necesidades de la víctima y ésta reciba ayuda podrían reducirse significativamente (Gracia y Herrero, 2006a; Loseke y Gelles, 1993; Muehlenhard y Kimes, 1999). Como apuntaron Gracia y Herrero (2006b): "son necesarios esfuerzos de educación pública que desafíen estas actitudes de tolerancia y transmitan la idea de responsabilidad social respecto a la violencia doméstica. Romper el clima social de tolerancia podría incrementar los costos para los agresores y contribuir al control social informal de la violencia doméstica contra la mujer" ( $p$. 128). Además de los esfuerzos de educación pública dirigidos a la población general, nuestros resultados sugieren que también son necesarias políticas públicas que ayuden a reducir la tolerancia hacia la violencia de pareja contra la mujer en población inmigrante en general y latinoamericana en particular. Estos esfuerzos de la educación pública necesitan transmitir la idea de que todas las formas de violencia son erróneas y no deben ser aceptadas bajo ninguna circunstancia.

Las actitudes de culpabilización de las víctimas también estuvieron asociadas con las actitudes hacia la denuncia entre inmigrantes latinoamericanos. Así, los participantes que culpabilizaron a las víctimas de la violencia tendieron a mostrar una actitud negativa hacia la denuncia. Culpabilizar a las víctimas no sólo implica tolerar la violencia bajo algunas circunstancias sino también reducir las posibilidades de las víctimas de recibir ayuda. De acuerdo con la teoría psicosocial, cuando se cree que las víctimas son causantes de sus propios problemas o que se lo merecen, las oportunidades de recibir ayuda se reducen significativamente (Batson, 1998; Gracia y Herrero, 2006b; Lerner 1970, 1980; Weiner, 1980, 1985). Estos resultados apuntan a la necesidad de dirigir esfuerzos de la educación pública hacia las frecuentes actitudes de culpabilización de las víctimas entre los inmigrantes latinoamericanos. Finalmente, aunque algunos estudios sugieren una relación entre conocimiento de víctimas y actitudes hacia la denuncia (e.g., Gracia y Herrero, 2006a), en este estudio encontramos que la exposición personal a incidentes de violencia de pareja no se relacionó con las actitudes hacia la denuncia.

Finalmente, el estudio tiene algunas limitaciones. En primer lugar, como ya mencionamos en la sección del método, de las tres muestras utilizadas en este estudio dos de ellas fueron probabilísticas y representativas de la población española mientras que la muestra de inmigrantes latinoamericanos no lo fue. En este sentido, deberíamos ser cautos a la hora de realizar comparaciones entre españoles e inmigrantes latinoamericanos acerca de sus visiones respectivas sobre la violencia de pareja. Sin embargo, la alta tasa de prevalencia de violencia de pareja entre inmigrantes latinoamericanos en España sugiere que las diferencias encontradas en nuestro estudio apuntan en la dirección correcta. En segundo lugar, no podemos generalizar los resultados a la población latinoamericana en España ya que nuestro estudio se ha focalizado en dos comunidades: Asturias y Valencia. Tampoco podemos descartar completamente la posibilidad de que alguno de los participantes inmigrantes en el estudio estuviera implicado directamente en algún caso de violencia en la pareja (como agresor o como víctima). De haber sido el caso, ello podría tener algún efecto en las relaciones encontradas en el estudio. Investigaciones centradas en los agresores podrían ser un contrapunto interesante para este estudio, permitiendo explorar en profundidad el papel que juegan las actitudes hacia la violencia de pareja y la exposición personal a la misma en la disposición a intervenir y a ejercer el control social informal en nuestras comunidades y vecindarios. La investigación futura en este campo debería atender a estas limitaciones potenciales.

\section{Referencias}

American Psychological Association Presidential Task Force on Violence and the Family. (1996). Report of the American Psychological Association Task Force on Violence and the Family. Washington, DC: Author.

Asbury, J. (1993). Violence in the families of color in the United States. En R. Hampton, T. Gullotta, G. Adams, E. Potter y R. Weissberg (Eds.), Family violence: Prevention and treatment (pp. 159-178). Newbury Park, CA: Sage.

Bachman, R. y Saltzman, L. E. (1995). Violence against 
women: Estimates from the redesigned survey. NCJ154348 Special Report. Washington, DC: U.S. Department of Justice, Bureau of Justice Statistics.

Batson, C. D. (1998). Altruism and prosocial behavior. En D. T. Gilbert, S. Fiske y G. Lindzey (Eds.), The handbook of social psychology (pp. 282-316). New York: McGrawHill.

Bennet, L. y Williams, O. J. (1999). Men who batter. En R. L. Hampton (Ed.), Family violence: Prevention and treatment (2 ${ }^{\text {nd }}$ Edition) (pp. 227-259). Thousand Oaks, CA: Sage.

Bennet, L. y Williams, O. J. (1999). Men who batter. En R. L. Hampton (Ed.), Family violence: Prevention and treatment (2 $2^{\text {nd }}$ Edition) (pp. 227-259). Thousand Oaks, CA: Sage.

Benson, M., Wooldredge, J., Thistlethwaite, A. y Fox, G. (2004). The correlation between race and domestic violence is confounded with community context. Social Problems, 51, 326-342.

Bhanot, S. y Senn, C. Y. (2007). Attitudes towards violence against women in men of south asian ancestry: Are acculturation and gender role attitudes important factors? Journal of Family Violence, 22, 25-31.

Biden, J. R. Jr. (1993). Violence against women: The congressional response. American Psychologist, 48, 10591061 .

Caetano, R., Cunradi, C. B., Clark, C. L. y Schafer, J. (2000). Intimate partner violence and drinking patterns among White, Black, and Hispanic couples in the U.S. Journal of Substance Abuse, 11, 123-138.

Campbell, D. W., Maskai, B. y Torres, S. (1997). Water on rock: Changing domestic violence perceptions in the African American, Asian American, and Latino communities. En E. Klein, J. Campbell, E. Soler y M. Guez (Eds.), Ending domestic violence: Changing public perceptions/halting the epidemic (pp. 64-87). Thousand Oaks, CA: Sage.

Council of Europe (2002). Recommendation Rec(2002)5 of the Committee of Ministers to member States on the protection of women against violence adopted on 30 April 2002 and Explanatory Memorandum. Strasbourg, France: Author.

Crowell, N. A. y Burgess, A. W. (1996). Understanding violence against women. Washington, DC: National Academy Press.

Dasgupta, S. D. (2000). Charting the course: An overview of domestic violence in the south Asian community in the United States. Journal of Social Distress and the Homeless, 9, 173-185.

Davis, R. C. y Erez, E. (1998). Immigrant population as victims: Toward a multicultural criminal justice system. Washington, DC: National Institute of Justice.

DeMaris, A. (1995). A tutorial in logistic regresión. Journal of Marriage and the Family, 57, 956-968.

European Social Survey (Retrieved February 20, 2008, from http://www.europeansocialsurvey.org/

Fagan, J. (1990). Contributions of research to criminal justice policy on wife assault. En D. Besharov (Ed.), Family violence: Research and public policy issues (pp. 53-81). Washington, D.C.: American Enterprise Institute Press.

Fagan, J. (1993). Social structure and spouse assault. En B. Forst (Ed.), The socio-economics of crime and justice (pp. 209-254). New York: Sharpe.

Fagan, J. A. (1989). Cessation of family violence: Deterrence and dissuassion. En M. Tonry y L. Ohlin (Eds.), Crime and justice: An annual review of research (pp. 377-425). Chicago: University of Chicago Press.

Ferrer, V. A., Bosch, E. y Riera, T. (2006). Las dificultades en la cuantificación de la violencia contra las mujeres en la pareja: análisis psicosocial. Intervención Psicosocial, 15, 181-201.

Gabler, M., Stern, S. E. y Miserandino, M. (1998). Latin American, Asian, and American cultural differences in perceptions of spousal abuse. Psychological Reports, 83, 587-592.

Gelles, R.J. (1983). An exchange/social control theory. En D. Finkelhor, R. J. Gelles, G. T. Hotaling y M. A. Straus (Eds.), The dark side of families (pp. 151-165). Thousand Oaks, CA: Sage.

Gelles, R.J. (1993). Through a sociological lens: Social structure and family violence. En R. J. Gelles y D. R. Loseke (Eds.), Current controversies on family violence (pp. 31-46). Newbury Park, CA: Sage.

Gondolf, E., Fisher, E. y McFerron, R. (1991). Racial differences among shelter residents: A comparison of Anglo, Black, and Hispanic battered women. En R. Hampton (Ed.), Black family violence: Current research and theory (pp. 103-113). Lexington, MA: Heath.

Goodman, L. A., Koss, M. P., Fitzgerald, L. F., Russo, N. F. y Keita, G. P. (1993). Male violence against women: Current research and future directions. American Psychologist, 48, 1054-1058.

Gracia, E. (2002). Visibilidad y tolerancia social de la violencia familiar. Intervención Psicosocial, 11, 5-15.

Gracia, E. (2004). Unreported cases of domestic violence against women: Towards an epidemiology of social silence, tolerance, and inhibition. Journal of Epidemiology and Community Health, 58, 536-537.

Gracia, E. y Herrero, J. (2006a). Public attitudes toward reporting partner violence against women and reporting behavior. Journal of Marriage and Family, 68, 759-768.

Gracia, E. y Herrero, J. (2006b). Acceptability of domestic violence against women in the European Union: A multilevel analysis. Journal of Epidemiology and Community Health, 60, 123-129.

Gracia, E. y Herrero, J. (2007). Perceived neighborhood social disorder and attitudes toward reporting domestic violence against women. Journal of Interpersonal Violence, 22; 737-752.

Gracia, E., García, F. y Lila, M. (2008). Police involvement in cases of intimate partner violence against women: The influence of perceived severity and personal responsibility. Violence Against Women, 14, 697-714.

Gracia, E., García, F. y Lila, M. (2009). Public responses to intimate partner violence against women: The influence of perceived severity and personal responsibility. The 
Spanish Journal of Psychology, 12, 648-656.

Gracia, E., Herrero, J., Lila, M. y Fuente, A. (2009). Perceived neighborhood social disorder and attitudes toward domestic violence against women among LatinAmerican immigrants. The European Journal of Psychology Applied to Legal Context, 1, 25-43.

Harden, B. J. y Koblinsky, S. A. (1999). Doble exposure: Children affected by family and community violence. En R. L. Hampton (Ed.), Family violence: Prevention and treatment ( $2^{\text {nd }}$ Edition) (pp. 66-102). Thousand Oaks, CA: Sage.

Hass, G. A., Dutton, M. A. y Orloff, L. E. (2000). Lifetime prevalence of violence against Latina immigrants: Legal and policy implications. International Review of Victimology, 7, 93-113.

Hazen, A. L. y Soriano, F. I. (2007). Experiences with intimate partner violence among latina women. Violence Against Women, 13, 562-582.

Herrero, J. y Gracia, E. (2005). Perceived frequency of domestic violence against women and neighbourhood social disorder. Psychological Reports, 97, 712-716.

Instituto de la Mujer (2003). La violencia contra las mujeres. Resultados de la macroencuesta. II Parte. Madrid, Spain: Author.

Instituto de la Mujer (2008). Denuncias por malos tratos producidas por el cónyuge o análogo. Año 2007. Madrid, Spain: Author.

Jasinski, J. L. (1998). The role of acculturation in wife assault. Hispanic Journal of Behavioral Sciences, 20, 175-192.

Jenkins, P. (1996). Threads that link community and family violence: Issues for prevention. En R. L. Hampton, P. Jenkins y T. P. Gullotta (Eds.), Preventing violence in America (pp. 33-52). London: Sage.

Kantor, G. K., Jasinski, J. L. y Aldarondo, E. (1994). Sociocultural status and incidence of marital violence in Hispanic families. Violence and Victims, 9, 207-222

Kelly, L. (1996). Tensions and possibilities: Enhancing informal responses to domestic violence. En J. L. Edleson y Z. C. Eisikovits (Eds.), Future interventions with battered women and their families (pp. 67-86). Thousand Oaks, CA: Sage.

Klein E., Campbell, J., Soler, E. y Ghez, M. (1997). Ending domestic violence: Changing public perceptions/halting the epidemic. Thousand Oaks, CA: Sage

Kury, H., Obergfell-Fuchs, J. y Woessner, G. (2003). The extent of family violence in Europe. Violence Against Women, 10, 749-769.

Lackey, C. y Williams, K. R. (1995). Social bonding and the cessation of partner violence across generations. Journal of Marriage and the Family, 57, 295-305.

Lerner, M. J. (1970). The desire for justice and reactions to victims. En J. Macaulay y L. Berkowitz (Eds.), Altruism and helping behavior (pp. 205-229). New York: Academic Press.

Lerner, M. J. (1980). The belief in a just world: A fundamental delusion. New York: Plenum.

Loseke, D. R. (1989). "Violence" is "violence"...or is it?
The social construction of "wife abuse" and public policy. En J. Best (Ed.), Images of issues: Typifying contemporary social problems (pp. 191-206). New York: de Gruyter.

Loseke, D. R. y Gelles, R.J. (1993). Conclusion: Social problems, social policy, and controversies on family violence. En R. J. Gelles y D. R. Loseke (Eds.), Current controversies on family violence (pp. 357-366). Newbury Park, CA: Sage.

McLoyd, V. C., Cauce, A. M., Takeuchi, D. y Wilson, L. (2000). Marital processes and parental socialization in families of color: A decade review of research. Journal of Marriage and Family, 62, 1070-1093.

Menard, S. (1995). Applied logistic regression analysis. Series: Quantitative applications in the social sciences. Thousand Oaks, CA: Sage.

Morash, M., Bui, H., Zhang, Y. y Holtfreter, K. (2007). Risk factors for abusive relationships: A study of Vietnamese American immigrant women. Violence Against Women, 13, 653-675.

Muehlenhard, C. L. y Kimes, L. A. (1999). The social construction of violence: The case of sexual and domestic violence. Personality and Social Psychology Review, 3, 234245.

Observatorio Permanente de la Inmigración (2009). Disponible en: http://extranjeros.mtin.es/es/InformacionEstadistica/Infor mes/Extranjeros30Septiembre2009/index.htmlr

Perilla, J. L., Bakeman, R. y Rorris, F. H. (1994). Culture and domestic violence: The ecology of abused Latinas. Violence and Victims, 9, 325-339.

Raj, A. y Silverman, J. G. (2002). Violence against immigrant women: The roles of culture, context, and legal immigrant status on intimate partner violence. Violence Against Women, 8, 367-398.

Rodriguez, R. (1998). Clinical interventions with battered migrant farm worker women. En J. C. Campbell (Ed.), Empowering survivors of abuse (pp. 271-279). Thousand Oaks, CA: Sage.

Sampson, R. J. y Lauritsen, J. L. (1994). Violent victimization and offending: Individual-, situational-, and community-level risk factors. En A. J. Reiss Jr. y J. Roth (Eds.), Understanding and preventing violence: Social Influences (Vol. 3, pp. 1-114). Washington, DC: National Academy Press.

Sorenson, S. B. y Telles, C. A. (1991). Self-reports of spousal violence in a Mexican-American and non-Latino White population. Violence and Victims, 6, 3-15.

Stowell J. I. y Martinez Jr., R. (2007). Comparing the context of immigrant homicides in Miami: Haitians, Jamaicans, and Mariels. Aggression and Violent Behavior, 12, 564-581.

Straus M. A. y Gelles, R. J. (1986). Societal change and change in family violence from 1974 to 1985 as revealed by two national surveys. Journal of Marriage and the Family, 48, 465-479.

Straus, M A., Gelles, R. A. y Steinmetz, S. K. (1980). Behind closed doors: Violence in the American family. Garden 
City, NY: Doubleday.

Tran, C. G. y Des Jardins, K. (2000). Domestic violence in Vietnamese refugee and Korean immigrant communities. En J. L. Chin (Ed.), Relationships among Asian American women (pp. 71-96). Washington, DC: American Psychological Asso-ciation

Van Hightower, N. R., Gorton, J. y DeMoss, C. L. (2000). Predictive models of domestic violence and fear of intimate partners among migrant and seasonal farm worker women. Journal of Family Violence, 15, 137-154.

Weiner, B. (1980). A cognitive (attribution)-emotion-action model of motivated behavior: an analysis of judgments of help giving. Journal of Personality and Social Psychology, 39, 186-200.
Weiner, B. (1995). Judgments of responsibility: A foundation for a theory of social conduct. New York: Guildford.

West, A. y Wandrei, M. L. (2002). Intimate partner violence: A model for predicting interventions by informal helpers. Journal of Interpersonal Violence, 17, 972-986.

West, C. M., Kantor, G. K. y Jasinski, J. L. (1998). Sociodemographic predictors and cultural barriers to help-seeking behavior by Latina and Anglo American battered women. Violence and Victims, 13, 361-375.

Wilson, W.J. (1987). The truly disadvantaged: The inner city, the underclass, and public policy. Chicago: University of Chicago Press.

World Health Organization (2002). World report on violence and health. Geneva, Switzerland: Author.

Manuscrito Recibido: 25/03/2010

Revisión Recibida: 03/06/2010

Manuscrito Aceptado: 03/06/2010 